\title{
Impact of Ceftiofur Administration in Steers on the Prevalence and Antimicrobial Resistance of Campylobacter spp.
}

\author{
Sicun Fan ${ }^{1}$, Derek Foster ${ }^{2}$, William G. Miller ${ }^{3}$, Jason Osborne ${ }^{4}$ and Sophia Kathariou ${ }^{1, *}$ \\ 1 Department of Food, Bioprocessing and Nutrition Sciences, North Carolina State University, \\ Raleigh, NC 27695, USA; sfan3@ncsu.edu \\ 2 Department of Population Health and Pathobiology, College of Veterinary Medicine, North Carolina State \\ University, Raleigh, NC 27695, USA; dmfoster@ncsu.edu \\ 3 Produce Safety and Microbiology Research Unit, Agricultural Research Service, U.S. Department of \\ Agriculture, Albany, CA 94710, USA; william.miller@usda.gov \\ 4 Department of Statistics, College of Sciences, North Carolina State University, Raleigh, NC 27695, USA; \\ jaosborn@ncsu.edu \\ * Correspondence: skathar@ncsu.edu; Tel.: +1-919-513-2075
}

Citation: Fan, S.; Foster, D.; Miller, W.G.; Osborne, J.; Kathariou, S. Impact of Ceftiofur Administration in Steers on the Prevalence and Antimicrobial Resistance of Campylobacter spp. Microorganisms 2021, 9, 318. https://doi.org/ 10.3390/microorganisms 9020318

Academic Editor:

Adolfo J. Martinez-Rodriguez

Received: 16 December 2020

Accepted: 1 February 2021

Published: 4 February 2021

Publisher's Note: MDPI stays neutral with regard to jurisdictional claims in published maps and institutional affiliations.

Copyright: (c) 2021 by the authors. Licensee MDPI, Basel, Switzerland. This article is an open access article distributed under the terms and conditions of the Creative Commons Attribution (CC BY) license (https:// creativecommons.org/licenses/by/ $4.0 /)$.

\begin{abstract}
Bacterial resistance to ceftiofur raises health concerns due to ceftiofur's extensive veterinary usage and structural similarity with the human antibiotic ceftriaxone. Ceftiofur crystalline-free acid (CCFA) and ceftiofur hydrochloride (CHCL) are ceftiofur types used therapeutically in cattle, but their potential impacts on Campylobacter prevalence and antimicrobial resistance remain unclear. In this study two groups of steers were each treated with CCFA or CHCL. In vivo active drug concentrations were measured and fecal samples were analyzed for Campylobacter for up to 42 days post-treatment. Following administration, the colonic concentration of ceftiofur initially increased then dropped to pre-treatment levels by day 8 . The estimated prevalence of Campylobacter spp. was significantly $(p=0.0009)$ higher during the first week after CCFA treatment than after CHCL treatment $(81.3 \%$ vs. 45.2\%). Campylobacter jejuni predominated overall, with other Campylobacter spp. mainly identified in the first week after CCFA treatment. No treatment impacts were noted on ceftiofur minimum inhibitory concentration (MIC) for C. jejuni (10-20 $\mu \mathrm{g} / \mathrm{mL}$ ). More C. jejuni genotypes were detected in CCFA-treated than CHCL-treated steers. These findings suggest that ceftiofur did not significantly impact Campylobacter prevalence or ceftiofur MIC. However, CHCL may be preferable due to the lower likelihood of temporary increases in Campylobacter prevalence.
\end{abstract}

Keywords: ceftiofur; CCFA; CHCL; cattle; Campylobacter; antibiotic resistance

\section{Introduction}

The use of antimicrobials in livestock can promote animal health and productivity [1], but also increases concerns related to emergence and persistence of antimicrobial resistance in zoonotic bacterial pathogens [2,3]. Among livestock, the highest domestic use of medically-important antimicrobials is in cattle (42\%) [4]. These antimicrobials include the 3rd-generation cephalosporins (3GCs) [5], which are also identified as the most critically-important antimicrobials for human medicine [6].

Ceftiofur is a 3GC that is exclusively used in veterinary medicine. In the United States, ceftiofur is one of the leading antimicrobials for feedlot cattle and lactating dairy cows, and is frequently employed therapeutically to treat respiratory disease in feedlot cattle and postpartum metritis in cows [7-9]. In addition, ceftiofur has been employed extra-label for the treatment of enteric disease in cattle [10], even though most extra-label administrations of cephalosporins were banned by the United States Food and Drug Administration in 2012 [11].

Major reasons for the common use of ceftiofur in beef and dairy cattle include its high effectiveness [12,13], zero withdrawal time for milk [14-16], and short withdrawal periods 
for meat $[15,17,18]$. However, ceftiofur is similar in structure to the antibiotic ceftriaxone which is used extensively in human medicine, and the two drugs have the same mode of action [19-21]. Concerns arise, therefore, that the use of ceftiofur for cattle conditions such as respiratory disease, metritis, and enteric disease can lead to ceftriaxone resistance in bacterial foodborne pathogens such as Salmonella enterica, Escherichia coli, and Campylobacter spp. These foodborne bacterial pathogens can inhabit the bovine gastrointestinal tract, typically without symptoms, and become transmitted to humans via the food chain $[21,22]$.

Injectable ceftiofur formulations in the market currently include ceftiofur crystallinefree acid (CCFA; Excede $\left.{ }^{\circledR}\right)$, ceftiofur hydrochloride $\left(\right.$ CHCL; Excenel $\left.{ }^{\circledR}\right)$, and ceftiofur sodium sterile powder (CSSP; Naxcel ${ }^{\circledR}$ ) [23]. These formulations have varied indications, approved dosages, routes of administration, and meat withdrawal periods [24]. In addition, they vary in pharmacokinetic parameters, including peak concentrations and half-life [25]. There is no clear differentiation among these formulations regarding treatment efficacy, and the choice is mainly based on convenience [17]. The potential impacts of different formulations of ceftiofur on zoonotic foodborne pathogens and their antimicrobial resistance remain poorly understood and in need of further investigation. Currently, only a few reports are available on the impact of ceftiofur treatment of cattle on the gut microbiome and antimicrobial resistance genes, and on specific enteric bacteria such as E. coli, Salmonella, and Enterococcus faecalis in the feces of the animals $[16,17,25,26]$.

Campylobacter spp. (family Campylobacteraceae) are Gram-negative, corkscrew-shaped bacteria that are highly motile and the only obligate microaerophiles among foodborne bacterial pathogens of humans [27]. The species most commonly implicated in human foodborne disease is Campylobacter jejuni followed by C. coli, and leading sources of transmission to humans include raw or undercooked poultry, raw milk, untreated water, and direct contact with animal feces [28]. Campylobacter, primarily C. jejuni, is a leading bacterial foodborne pathogen in the United States and other nations [29]. Common outcomes of campylobacteriosis include acute gastroenteritis characterized by diarrhea and severe abdominal pain, and campylobacteriosis is also the leading antecedent for the severe autoimmune disease Guillain-Barré syndrome [30]. Campylobacteriosis is typically a selflimited disease and treatment, when mandated, involves fluoroquinolones or macrolides, with the latter being increasingly preferred due to the high prevalence of fluoroquinolone resistance in C. jejuni and C. coli [31,32].

Campylobacter is routinely found in cattle and typically carried asymptomatically $[27,33,34]$. However, Campylobacter from bovine feces can contaminate milk, water or meat during processing [34]. Previous studies identified exposure to cattle and raw ground beef as risk factors for human campylobacteriosis $[35,36]$. In addition, numerous outbreaks of campylobacteriosis have been attributed to consumption of raw milk [37-39]. Furthermore, drug-resistant Campylobacter is listed among the "serious threats" in the most recent Antimicrobial Resistance Threats Report by the Centers for Disease Control and Prevention [32]. As discussed above, ceftiofur is frequently used therapeutically in dairy and beef cattle [7-10]. However, currently little is known regarding the potential impacts of ceftiofur treatment on the prevalence and antimicrobial resistance of Campylobacter in cattle.

In a previous study, we monitored the intestinal concentrations of CCFA and CHCL after treatment of steers with these drugs and investigated the impact of the treatment on microbiome composition as well as on the prevalence and minimum inhibitory concentration (MIC) of ceftiofur of E. coli in the feces of the animals [17]. We noted shifts in microbiome composition and decreased E. coli levels, especially in the CCFA-treated group, as well as temporary increases in the MIC of ceftiofur in both treatment groups with MIC values peaking at 96 and $48 \mathrm{~h}$ following the CCFA and CHCL treatment, respectively [17]. In the current study, our objective was to investigate the impact of CCFA (sustained but lower intestinal drug concentrations) and CHCL (shorter duration but higher intestinal drug concentrations) treatment of these steers on Campylobacter prevalence, tolerance to ceftiofur and other antimicrobials and multilocus sequence typing (MLST)-based genotypes. We hypothesized that the treatment impact may differ between cattle receiving 
CCFA and those receiving CHCL. Fecal samples were analyzed for Campylobacter before and after treatment in the two separate groups of steers that were administered CCFA and CHCL, respectively, and Campylobacter prevalence was assessed in the context of the drug concentration levels in the colon.

\section{Materials and Methods}

\subsection{Steers and Treatment}

The steers for this study and their treatment have been previously described [17]. They consisted of 12 healthy Holstein steers (six months old and weighing 186-288 kg) and were obtained from the Dairy Educational Unit at North Carolina State University (NCSU) in Raleigh, NC, USA. They were housed in pairs in stalls at the College of Veterinary Medicine (CVM), NCSU, from May 2016 to July 2016, as described in [17]. The steers for the CCFA treatment $(n=6)$ were here designated D1-D6 while those for CHCL $(n=6)$ were designated N1-N6. The CCFA treatment study took place from May 18 through 8 June 2016 for steers D1, D2, D3, and D4 and from 1 June through 13 July 2016 for steers D5 and D6. The CHCL treatment was from 24 June through 12 July 2016 for steers N1, N2, N3, and N4 and from June 29 through 12 July 2016 for steers N5 and N6. As previously described [17], the sample size was determined based on previous pharmacokinetic-pharmacodynamic studies in cattle $[25,40,41]$. All steers received lidocaine and flunixin meglumine for disbudding within the first month of life, while steer D6 received flunixin and CHCL for four days due to diarrhea and fever, several months prior to the CCFA treatment.

The ceftiofur treatments of the steers were previously described [17]. Briefly, ultrafiltration probes for drug level determination were placed in the ileum and spiral colon as described [17]. Within 24-48 h after placement of the probes, the steers were administered either CCFA (6.6 mg/kg, one dose; Zoetis, Parsippany, NJ, USA) or CHCL (2.2 mg/kg, three daily doses; Zoetis). Both drugs were injected subcutaneously, either at the base of the ear (CCFA) or in the neck (CHCL) [17]. This study was conducted under the approval of the NCSU Institutional Animal Care and Use Committee (protocol \# 18-020A).

\subsection{Gastrointestinal (GI) Fluid Collection, Active Drug Concentration Determination, and Pharmacokinetic Analysis}

The drug concentration levels in the ileum and spiral colon were determined as described before, and have been previously reported [17]. Briefly, the active drug concentration in the intestinal fluid was measured using reverse-phase high pressure liquid chromatography with ultraviolet detection $[17,25,42]$. The pharmacokinetic analysis was performed using standard methods and the pharmacokinetic parameters were calculated by a pharmacokinetic computer program (Phoenix, V. 8.0; Pharsight Corporation, Certara, St. Louis, MO, USA) as described [17]. As previously indicated, for CCFA the fluid samples were collected immediately prior to treatment (time 0 ) and at 2, 4, 8, 12, 24, 32, 48, 72, 96, $120,144,168$, and $192 \mathrm{~h}$ after treatment; for CHCL the collecting points were immediately prior to treatment (time 0 ) and at 2, 4, 6, 8, 12, 24, 26, 28, 30, 32, 36, 48, 50, 52, 54, 56, 60, 72, 74,78 , and $96 \mathrm{~h}$ after the first dose [17].

\subsection{Sample Collection and Processing for Campylobacter}

Fecal samples (hereafter frequently referred to as "samples") were collected using sterile lubricant and a clean rectal sleeve as described [17,25,41]. The sampling points were largely similar for both treatment groups: $0 \mathrm{~h}$ (i.e., immediately before treatment), every 12 $\mathrm{h}$ until $72 \mathrm{~h}$ post-treatment, and daily thereafter until day 8 , with samples also collected at less regular intervals thereafter up until day 35-42 for CCFA steers, and day 13-18 for the CHCL group (Table S1). The collected samples were packed individually, delivered to the laboratory overnight on ice and processed upon arrival.

To isolate Campylobacter, fecal samples were processed as described [43], unless stated otherwise. Specifically, $100 \mathrm{mg}$ of each sample was added to $2 \mathrm{~mL}$ of Bolton broth (Oxoid Ltd., Basingstoke, Hampshire, UK), containing the corresponding supplement 
(SR183E, Oxoid) and laked horse blood (SR048C, Oxoid), in sterile 15-mL polypropylene Corning tubes (Fisher Scientific, Pittsburgh, PA, USA). The tubes were vortexed gently, covered loosely with caps, and incubated microaerobically at $37^{\circ} \mathrm{C}$ overnight in anaerobic jars with GasPak EZ CampyPak container system sachets (Becton Dickinson, Sparks, MD, USA). Then, $100 \mu \mathrm{L}$ of the overnight enrichment was serially diluted with sterile Mueller-Hinton broth (MHB; Difco, Becton Dickinson), plated (100 $\mu \mathrm{L})$ onto modified charcoal-cefoperazone-deoxycholate agar (mCCDA; Oxoid) containing the corresponding supplement (SR0155E, Oxoid), and incubated microaerobically for $48 \mathrm{~h}$ at $37^{\circ} \mathrm{C}$. For a set of samples from the CCFA group (steers D1, D2, D3, and D4) the mCCDA plates were also incubated microaerobically at $42{ }^{\circ} \mathrm{C}$ for $48 \mathrm{~h}$. Under the assumption that even one CFU in the original sample $(100 \mathrm{mg})$ would yield a positive result after enrichment and plating, the limit of detection for Campylobacter was $10 \mathrm{CFU} / \mathrm{g}$ of feces.

$\mathrm{Up}$ to seven colonies per positive sample were chosen randomly from the mCCDA plates at $37^{\circ} \mathrm{C}$ and purified under microaerobic conditions on Mueller-Hinton agar incubated at $37^{\circ} \mathrm{C}$ for $48 \mathrm{~h}$ (MHA; Difco, Becton Dickinson; Table S1). Colonies from those mCCDA plates that had been incubated at $42{ }^{\circ} \mathrm{C}$ were purified on MHA following incubation at $42^{\circ} \mathrm{C}$ for $48 \mathrm{~h}$. A culture derived from a single colony is hereafter frequently referred to as a Campylobacter "isolate". Pure cultures were preserved at $-80^{\circ} \mathrm{C}$ in brain heart infusion broth (BHI; Becton Dickinson) with 20\% sterile glycerol. Campylobacter recovery and isolates from each animal and time point are listed in Table S1.

\subsection{Campylobacter Species Determination, Multilocus Sequence Typing (MLST), and Minimum Spanning Tree (MST)}

Genomic DNA was extracted from pure Campylobacter cultures using the DNeasy Blood and Tissue Kit (Qiagen, Valencia, CA, USA) following the manufacturer's protocol. Multiplex PCR was performed using the hipO and ceuE primers to identify C. jejuni and C. coli, respectively, as described [44]. Campylobacter isolates that were negative for C. jejuni or $C$. coli were tested via multiplex PCR using sapB2 and $23 S$ rRNA primers specific for $C$. fetus and Campylobacter spp., respectively, as described [45], with the minor modification of extension at $72{ }^{\circ} \mathrm{C}$ for $1 \mathrm{~min}$. Campylobacter isolates that were PCR-negative for C. jejuni, C. coli or $C$. fetus were analyzed by MLST as described below and found to be $C$. hyointestinalis based on the C. fetus/C. hyointestinalis MLST scheme [46].

MLST analysis was performed for 67 isolates as described [46,47]. For each steer, isolates were chosen to represent the first and last instance of encountering a specific species-antimicrobial resistance (AMR) profile combination, except for combinations which were encountered only once and were therefore represented by a single isolate (Table S2). The sequence type (ST) of C. jejuni D3-5d-42-A was determined in silico upon analysis of whole genome sequence data that were available for this strain through another study, via Sequence Query, PubMLST (https:/ / pubmlst.org/bigsdb?db=pubmlst_campylobacter_ seqdef) (Table S2). Novel STs were submitted to the C. jejuni/C. coli MLST database (http://pubmlst.org/campylobacter/) for ST assignment. The relationships among the STs are displayed by a minimum spanning tree (MST) constructed using BioNumerics (version 7.6.3) as described [47].

\subsection{Campylobacter Antimicrobial Susceptibility and MIC Determinations}

All isolates were routinely tested for resistance to a panel of antimicrobial agents (tetracycline, streptomycin, nalidixic acid, ciprofloxacin, erythromycin, kanamycin, and gentamicin) as described [48,49], using the pan-sensitive (PS) C. jejuni ATCC 33560 as a quality assurance control. To assess potential changes of the minimum inhibitory concentration (MIC) of ceftiofur over time, 25 C. jejuni isolates were selected from the CCFA $(n=19)$ and CHCL $(n=6)$ groups (Table S3) based on the following criteria: they originated from the same steer at the same incubation temperature, had the same species and AMR combination and the same or closely-related ST, and were isolated at different times. In addition to ceftiofur $(1,10,20$, and $30 \mu \mathrm{g} / \mathrm{mL}$ ), this panel was tested for MICs of tetracycline (1 to 
$128 \mu \mathrm{g} / \mathrm{mL}$ ), kanamycin (1 to $256 \mu \mathrm{g} / \mathrm{mL}$ ), nalidixic acid (1 to $128 \mu \mathrm{g} / \mathrm{mL}$ ), ciprofloxacin (1 to $32 \mu \mathrm{g} / \mathrm{mL}$ ), ampicillin (1 to $32 \mu \mathrm{g} / \mathrm{mL}$ ), and enrofloxacin (1 to $32 \mu \mathrm{g} / \mathrm{mL}$ ).

\subsection{Statistical Analysis}

To estimate the effect of incubation temperature on the prevalence of $C$. jejuni from cattle in the CCFA treatment group, a generalized linear mixed model was fit using the GLIMMIX procedure of SAS version 9.4 (SAS Institute, Cary, NC, USA). The binary response variable was the presence (yes or no) of $C$. jejuni at each sampling time from $0 \mathrm{~h}$ (i.e., before treatment) to 8 days post-treatment from CCFA steers D1, D2, D3, and D4 with the explanatory variable being the incubation temperature $\left(37^{\circ} \mathrm{C}\right.$ or $\left.42{ }^{\circ} \mathrm{C}\right)$. Individual cattle and sampling time were considered as random effects to account for the interventions. The estimated prevalence of $C$. jejuni was derived from this model using SAS 9.4. The same statistical analysis was also performed for the non-C. jejuni, non-C. coli isolates.

To visualize the prevalence of $C$. jejuni per sampling point in the CCFA (steers D1, D2, $\mathrm{D} 3$, and D4) and CHCL treatment groups (steers N1, N2, N3, N4, N5, and N6) at $37^{\circ} \mathrm{C}$, the statements PROC MEANS and PROC GPLOT (SAS 9.4) were used. By visually checking the graphical distribution, the sampling time points were grouped into week 0 (immediately before treatment, $0 \mathrm{~h}$ ), week 1 ( $12 \mathrm{~h}$ to day 7 ), and week 2 (day 8 ). To estimate the effect of the treatment type (CCFA or CHCL) on the prevalence of $C$. jejuni, i.e., the estimated prevalence at different weeks, a generalized linear mixed model with binomial response was fit by the GLIMMIX procedure in SAS 9.4. The response variable was the clustered prevalence of $C$. jejuni at each week from week 0 to week 2; the explanatory variables were the treatment type, week, and their interaction. Variability among individual cattle was modeled by including random effects for cattle. The estimated prevalence of $C$. jejuni and all detected Campylobacter spp., i.e., combining C. jejuni, C. fetus, and C. hyointestinalis, was derived from this model using SAS 9.4. Statistical significance was set at $p$-value 0.05 .

\section{Results}

\subsection{Impacts of CCFA and CHCL on Prevalence of Campylobacter spp.}

A total of 168 fecal samples, 80 and 88 from the CCFA and the CHCL group, respectively, were analyzed for Campylobacter spp. At pre-treatment (time 0), three of the six steers in each treatment group were found to be Campylobacter-positive. For the CCFA samples, two fecal samples (one/steer; steers D2 and D3) were Campylobacter-positive at both $37^{\circ} \mathrm{C}$ and $42{ }^{\circ} \mathrm{C}$ and yielded C. jejuni, while the third (steer D4) was only positive at $37^{\circ} \mathrm{C}$ and yielded C. fetus (Table S1). Similar findings were obtained for the samples from the CHCL group, which were processed only at $37^{\circ} \mathrm{C}$. Specifically, of the six steers in this group three (N4, N5, N6) were Campylobacter-positive and yielded C. jejuni (Table S1). However, all 12 steers were positive for Campylobacter post-treatment (Table S1). The prevalence of Campylobacter-positive samples post-treatment varied by temperature in the CCFA group, being higher at $42{ }^{\circ} \mathrm{C}(42 / 52,80.8 \%)$ than at $37^{\circ} \mathrm{C}(44 / 66,66.7 \%)$ (Figure 1A). As indicated above, the CHCL samples were only examined at $37^{\circ} \mathrm{C}$, and 26 of the 82 post-treatment samples (31.7\%) were Campylobacter-positive (Figure 1B). Due to the apparent impact of temperature on prevalence that was observed with the CCFA samples, the comparative assessment of the impact of CCFA vs. CHCL treatment on Campylobacter was assessed at the common temperature of $37^{\circ} \mathrm{C}$. 

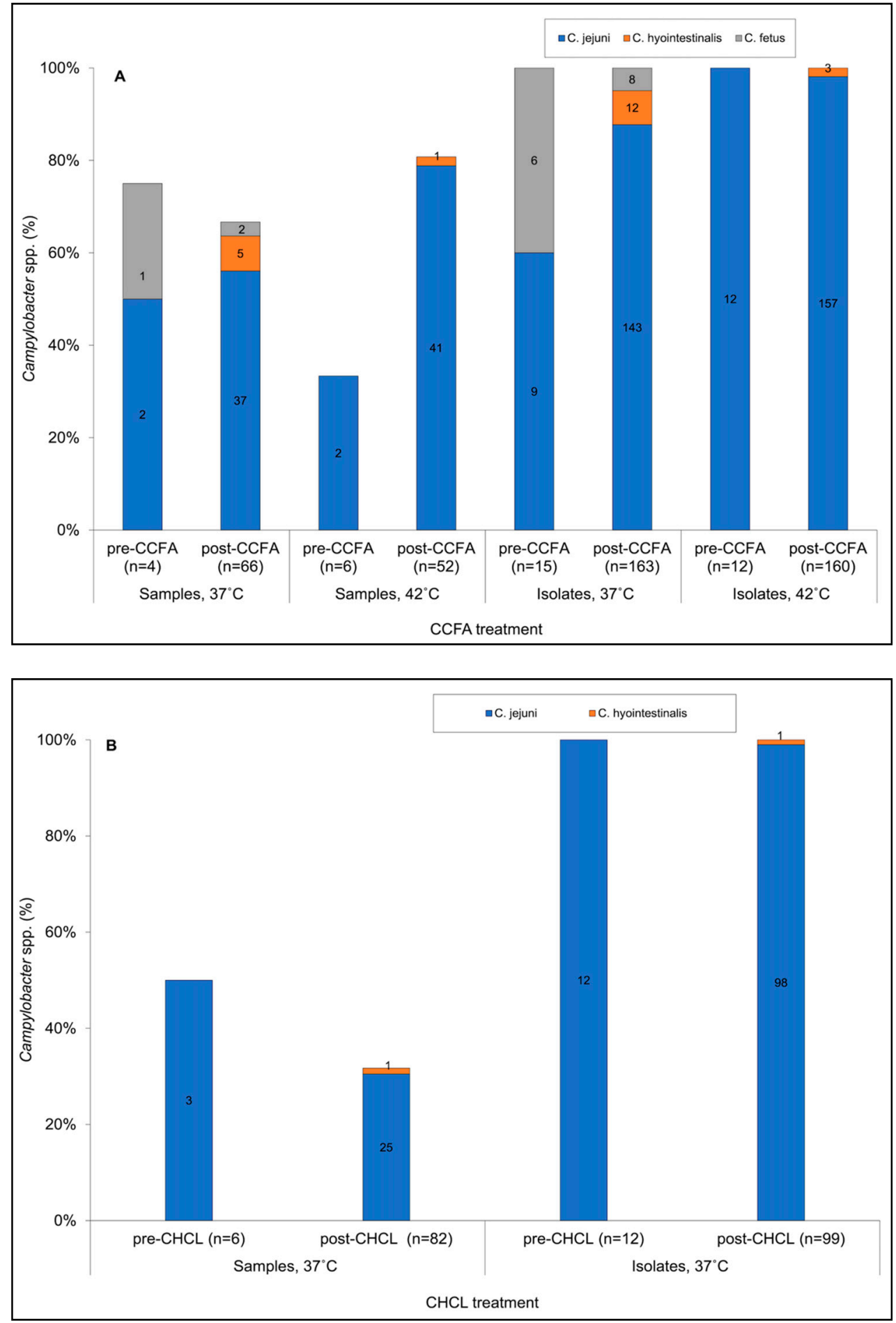

Figure 1. Prevalence of the different Campylobacter species in fecal samples from (A) ceftiofur crystalline-free acid (CCFA) and (B) ceftiofur hydrochloride (CHCL) groups. Campylobacter species designations are as in the inset, with blue for C. jejuni, orange for $C$. hyointestinalis, and gray for $C$. fetus. Pre-CCFA or pre-CHCL correspond to data pre-treatment (time 0 ), while post-CCFA and post-CHCL correspond to the compilation of all data post-treatment. "Samples" refers to fecal samples collected at different time points from the steers, while "Isolates" refers to Campylobacter cultures that were recovered from the fecal samples following enrichment and single colony purification. The four panels shown for the CCFA group in (A) correspond to, from left to right, (1), data from all fecal samples following incubation at $37{ }^{\circ} \mathrm{C}$; (2), data from all fecal samples following incubation at $42{ }^{\circ} \mathrm{C}$; (3), data from all Campylobacter isolates obtained following incubation at $37^{\circ} \mathrm{C}$; and (4), data from all Campylobacter isolates obtained following incubation at $42{ }^{\circ} \mathrm{C}$. The two groups shown for the $\mathrm{CHCL}$ group in (B) correspond to data from all fecal samples (left) and data from all Campylobacter isolates (right). CHCL samples were only incubated at $37^{\circ} \mathrm{C}$. Campylobacter detection and species determinations were as described in Materials and Methods. 
In the CHCL group the estimated prevalence of Campylobacter-positive samples was higher pre-treatment than one week post-treatment (57.5\% vs. $45.2 \%$ : Figure $2 \mathrm{~A}$ ), but significant immediate impacts of treatment were not noted in either group. On the other hand, cattle treated with CCFA had a significantly higher estimated prevalence of Campylobacter at week 1 vs. week $2(81.3 \%$ vs. $29.2 \% ; p=0.0108)$, while the differences did not achieve similar significance for the CHCL group $(p=0.0689)$ (Figure 2A). Furthermore, even though the estimated prevalence of Campylobacter did not differ significantly between the two groups pre-treatment, at week 1 post-treatment it was significantly higher in the CCFA group $(p=0.0009)$ (Figure 2A).
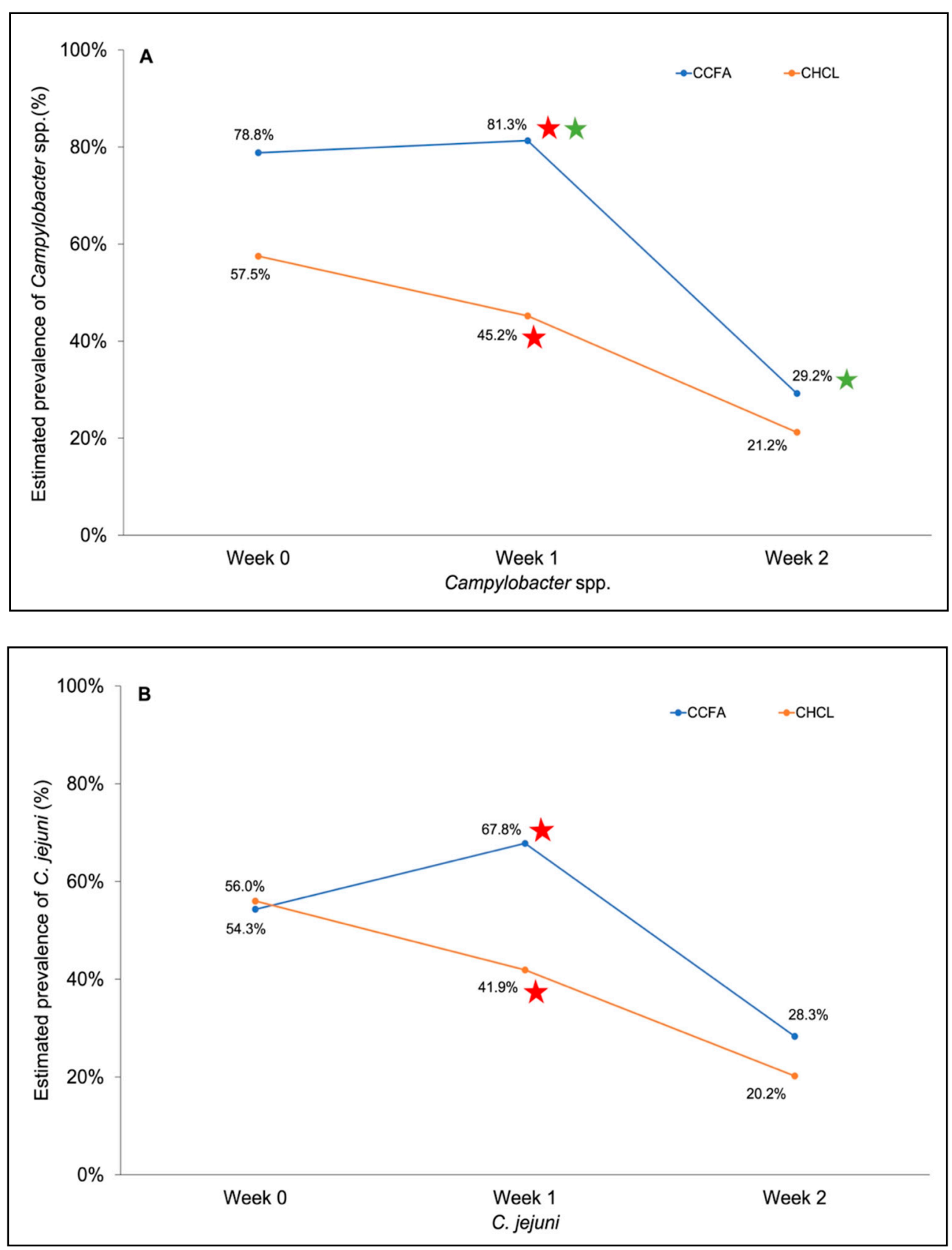

Figure 2. Estimated prevalence of (A) total Campylobacter spp. and (B) C. jejuni from the CCFA and CHCL groups. Red stars indicate a significant difference of estimated prevalence of Campylobacter spp. $(p=0.0009)$ or C. jejuni $(p=0.0184)$ between the CCFA and CHCL groups. Green stars indicate a significant difference $(p=0.0108)$ of the estimated prevalence of Campylobacter spp. between week 1 and 2 under CCFA treatment. Weeks 0,1 and 2 correspond to pre-treatment $(0 \mathrm{~h}), 12 \mathrm{~h}$ to day 7, and day 8, respectively. Data are based on CCFA and CHCL samples processed at $37{ }^{\circ} \mathrm{C}$. Estimated prevalence determinations were performed as described in Materials and Methods. 
Regardless of treatment group, the majority of the Campylobacter-positive samples yielded C. jejuni (Figure 1), and the estimated prevalence of C. jejuni largely reflected the trends described above for total Campylobacter-positive samples. It was similar between CCFA and CHCL samples prior to treatment (54.3\% vs. 56.0\%), but in week 1 after treatment it was significantly higher in CCFA than CHCL (67.8\% vs. $41.9 \% ; p=0.0184$; Figure 2B). CCFA samples also had a nearly-significant $(p=0.062)$ higher estimated prevalence of $C$. jejuni at week 1 vs. week $2(67.8 \%$ vs. $28.3 \%)$ while for CHCL the estimated prevalence was more stable (Figure 2B).

\subsection{Incubation Temperature Impacted the Estimated Prevalence of C. jejuni and Other Campylobacter spp.}

As indicated above, the majority of the Campylobacter-positive samples from either CCFA or CHCL steers yielded C. jejuni (Figure 1). For those CCFA samples that were processed at both $37{ }^{\circ} \mathrm{C}$ and $42{ }^{\circ} \mathrm{C}$, the estimated prevalence of $\mathrm{C}$. jejuni was significantly higher at $42{ }^{\circ} \mathrm{C}(p=0.0435 ; 79.7 \%$ vs. $59.5 \%)$. C. coli was not isolated from any of the samples, while other Campylobacter spp. were encountered only occasionally, and mostly in the CCFA samples at $37^{\circ} \mathrm{C}$ (Figure 1). Interestingly, C. hyointestinalis was detected in the CCFA samples only post-treatment and was recovered from $7.6 \%$ and $1.9 \%$ of the post-treatment samples at $37^{\circ} \mathrm{C}$ and $42{ }^{\circ} \mathrm{C}$, respectively (Figure 1A), while in the CHCL group C. hyointestinalis was detected only once, again post-treatment (Figure 1B). C. fetus was identified only in the CCFA samples at $37^{\circ} \mathrm{C}$, with higher prevalence pre-treatment than post-treatment (25\% vs. 3.0\%; Figure 1A). Surprisingly, C. fetus was not detected in the CHCL group at any time, even though $37{ }^{\circ} \mathrm{C}$ was employed for Campylobacter isolation and, as mentioned above, C. hyointestinalis was only encountered once in this group. Even though the infrequent recovery of non-C. jejuni Campylobacter spp. prevented significance assessments, the collective data suggest that their recovery was favored at $37^{\circ} \mathrm{C}$ post-treatment.

3.3. The Prevalence of Campylobacter-Positive Samples Reflected the Drug Concentration in the Colon, But C. jejuni Ceftiofur MIC Was Not Impacted by Either Treatment

Drug concentrations in the colon were monitored until day 8 post-treatment. The prevalence of $C$. jejuni during this time largely followed the trends in drug concentration. In the CCFA group, the drug concentration increased following administration and eventually dropped to pre-treatment levels by day 8, while in the CHCL group the levels reached three peaks at 6,30 , and $56 \mathrm{~h}$, corresponding to the repeated administration of the drug, and then dropped to the pre-treatment levels by day 8 (Figure 3). The half-life of CCFA in the colon was approximately 2-fold longer (94.85 h) than CHCL (39.45 h) [17]. The prevalence of total Campylobacter spp. was similar to C. jejuni except at 12 and $24 \mathrm{~h}$ post-treatment in the CCFA group, when most samples positive for $C$. fetus and C. hyointestinalis were identified in the CCFA-treated animals (Figure 3).

A panel of $C$. jejuni representing different time points prior to and subsequent to CCFA and CHCL treatment were examined for their tolerance to ceftiofur. Isolates were chosen from those steers that had yielded C. jejuni prior to treatment, i.e., at time 0 (Table S1). No impact of treatment on ceftiofur MIC could be noted among any of the isolates (Tables S1 and S3). MIC varied somewhat by animal $(10 \mu \mathrm{g} / \mathrm{mL}$ for isolates from D2 vs. $20 \mu \mathrm{g} / \mathrm{mL}$ for those from D3, N5, and N6), but the levels were the same regardless of time before and after treatment. In addition, for the tested isolates from the CCFA group, the MIC was the same for isolates from the same animal regardless of whether they were obtained at $37^{\circ} \mathrm{C}$ or $42{ }^{\circ} \mathrm{C}$ (Tables S1 and S3). Similar to ceftiofur, examination of a panel of other antimicrobials (e.g., nalidixic acid, kanamycin, ciprofloxacin, and enrofloxacin) also failed to reveal consistent impacts of treatment on MICs (Table S3). 


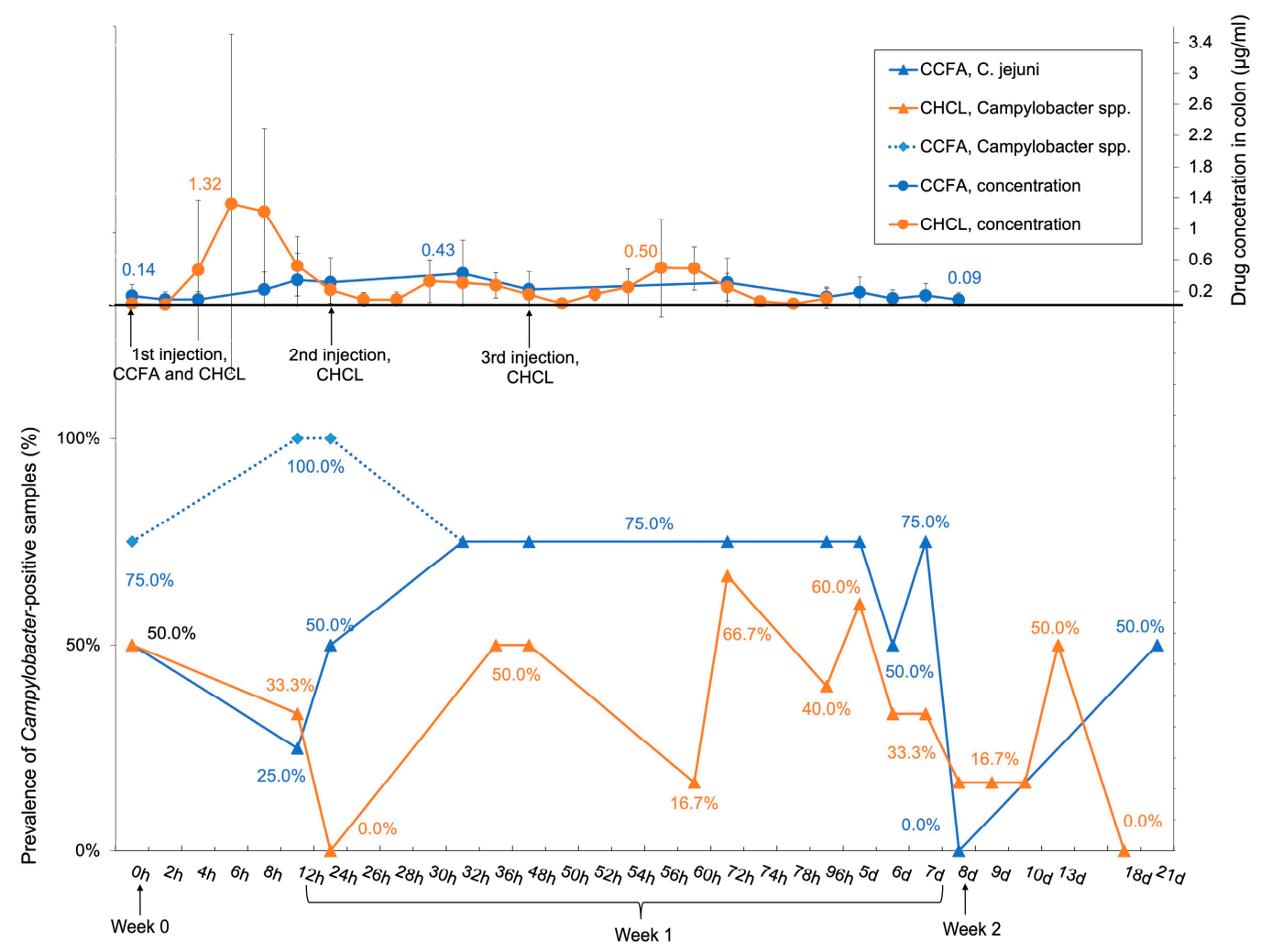

Figure 3. Drug concentrations in the colon and prevalence of Campylobacter-positive samples from the CCFA and CHCL groups. Upper panel, concentrations of CCFA (blue) and CHCL (orange). These drug concentration data were also presented earlier [17]. Lower panel, prevalence of Campylobacter spp. in CCFA (blue dashed lines) and CHCL (solid orange lines). Solid blue lines indicate prevalence of $C$. jejuni in the CCFA samples. C. jejuni prevalence in CHCL is not shown, as it was almost identical to total Campylobacter spp. Prevalence data are based on CCFA and CHCL samples processed at $37^{\circ} \mathrm{C}$. The concentrations of the drugs in the colon and prevalence of Campylobacter spp. and C. jejuni were determined as described in Materials and Methods.

3.4. Isolates Resistant to Both Tetracycline and Kanamycin Predominated Pre-and Post-Treatment in BOTH Treatment Groups

Testing the C. jejuni isolates with a panel of seven antimicrobials other than ceftiofur, specifically tetracycline, streptomycin, nalidixic acid, ciprofloxacin, erythromycin, kanamycin, and gentamicin, revealed just two AMR profiles: pan-susceptible (PS) and with resistance to only tetracycline and kanamycin (TK) (Table S1 and Figure 4). Ceftiofur MICs did not appear to vary between C. jejuni PS and C. jejuni TK isolates, with an MIC of $20 \mu \mathrm{g} / \mathrm{mL}$ being determined for both AMR profiles (Tables S1 and S3). C. jejuni TK isolates were encountered in each treatment group, both pre- and post-treatment. In the CCFA group, for which isolates were obtained at both $37{ }^{\circ} \mathrm{C}$ and $42{ }^{\circ} \mathrm{C}$, the use of the higher temperature $\left(42^{\circ} \mathrm{C}\right)$ appeared to favor the detection of samples positive for $\mathrm{C}$. jejuni TK (Figure 4A). Therefore, the potential impact of CCFA vs. CHCL treatment on recovery of $C$. jejuni TK was assessed at the common temperature of $37^{\circ} \mathrm{C}$. These comparisons suggested that the prevalence of $C$. jejuni TK-positive samples was similar before and after treatment in the CCFA group (Figure 4A), while it was higher post-treatment in the CHCL samples (Figure 4B). However, the pre- and post-treatment differences in the CHCL group were not statistically assessed due to a lack of power from limited samples and isolates pre-treatment. 

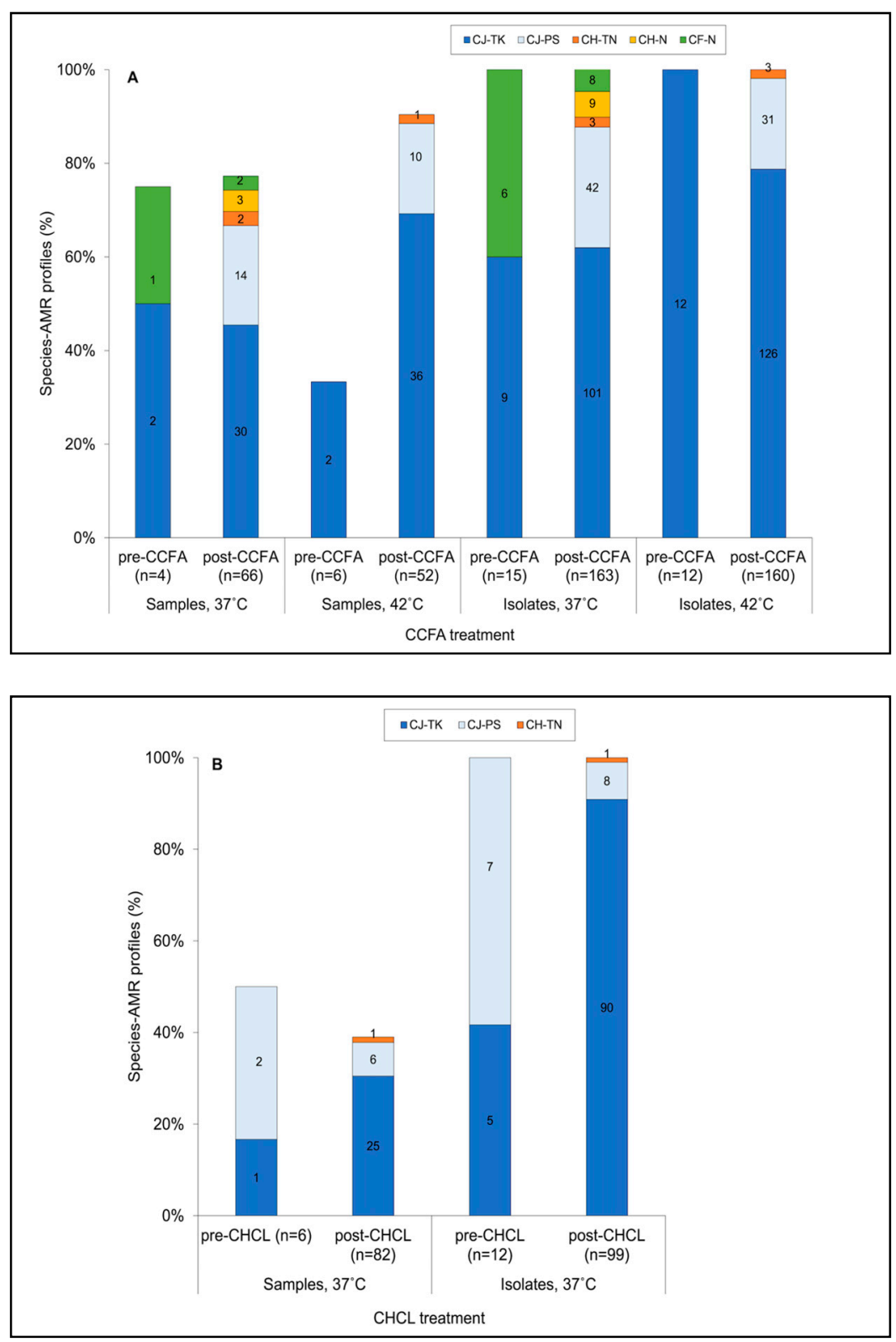

Figure 4. Prevalence of different species and antimicrobial resistance (AMR) profiles in Campylobacter samples and isolates from (A) CCFA and (B) CHCL groups. Species-AMR combinations are as in inset where $\mathrm{CJ}, \mathrm{CH}$, and $\mathrm{CF}$ indicate C. jejuni, C. hyointestinalis, and C. fetus, respectively. AMR abbreviations: TK, resistance only to tetracycline and kanamycin; PS, pan-susceptible; TN, resistance only to tetracycline and nalidixic acid; N, resistance only to nalidixic acid. Pre-CHCL or -CCFA referred to $0 \mathrm{~h}$, while post-CHCL or -CCFA was a compilation of all sampling points after $0 \mathrm{~h}$. A total of 12 samples from CCFA (seven at $37^{\circ} \mathrm{C}$ and five at $42^{\circ} \mathrm{C}$, all post-treatment) and six from CHCL (post-treatment) yielded both C. jejuni TK and C. jejuni PS. "Samples" and "Isolates" are defined as described in the legend of Figure 1. Campylobacter detection from the fecal samples and determination of species and AMR profiles were as described in the Materials and Methods.

It was noted that AMR profiles differed between C. jejuni and the other Campylobacter species. While C. jejuni isolates were either TK or PS, C. hyointestinalis were resistant either to tetracycline and nalidixic acid (TN) or only to nalidixic acid (N), and C. fetus were only resistant to nalidixic acid (N) (Figure 4). Samples positive for $C$. hyointestinalis TN were found only post-treatment, with similar prevalence (around 2.0\%) in CCFA and CHCL 
samples, while $C$. hyointestinalis $\mathrm{N}$ was only detected in three post-treatment samples of the CCFA group (Figure 4).

3.5. Multilocus Sequence Typing (MLST) Analysis Suggests that CCFA Treatment May Favor the Diversity of C. jejuni

As indicated earlier, the sequence type (ST) of a panel of 68 isolates was determined with 50 from the CCFA group and 18 from the CHCL group. These isolates represented, for each treatment, the first as well as (when applicable) the last date of each species-AMR combination (Table S2). The panel included 60 C. jejuni with 10 MLST-based STs (Figure 5) as well as six C. hyointestinalis with two STs and two C. fetus, both of the same ST (Table S2). Of the 10 C. jejuni STs, 7 were novel (Table S2 and Figure 5).

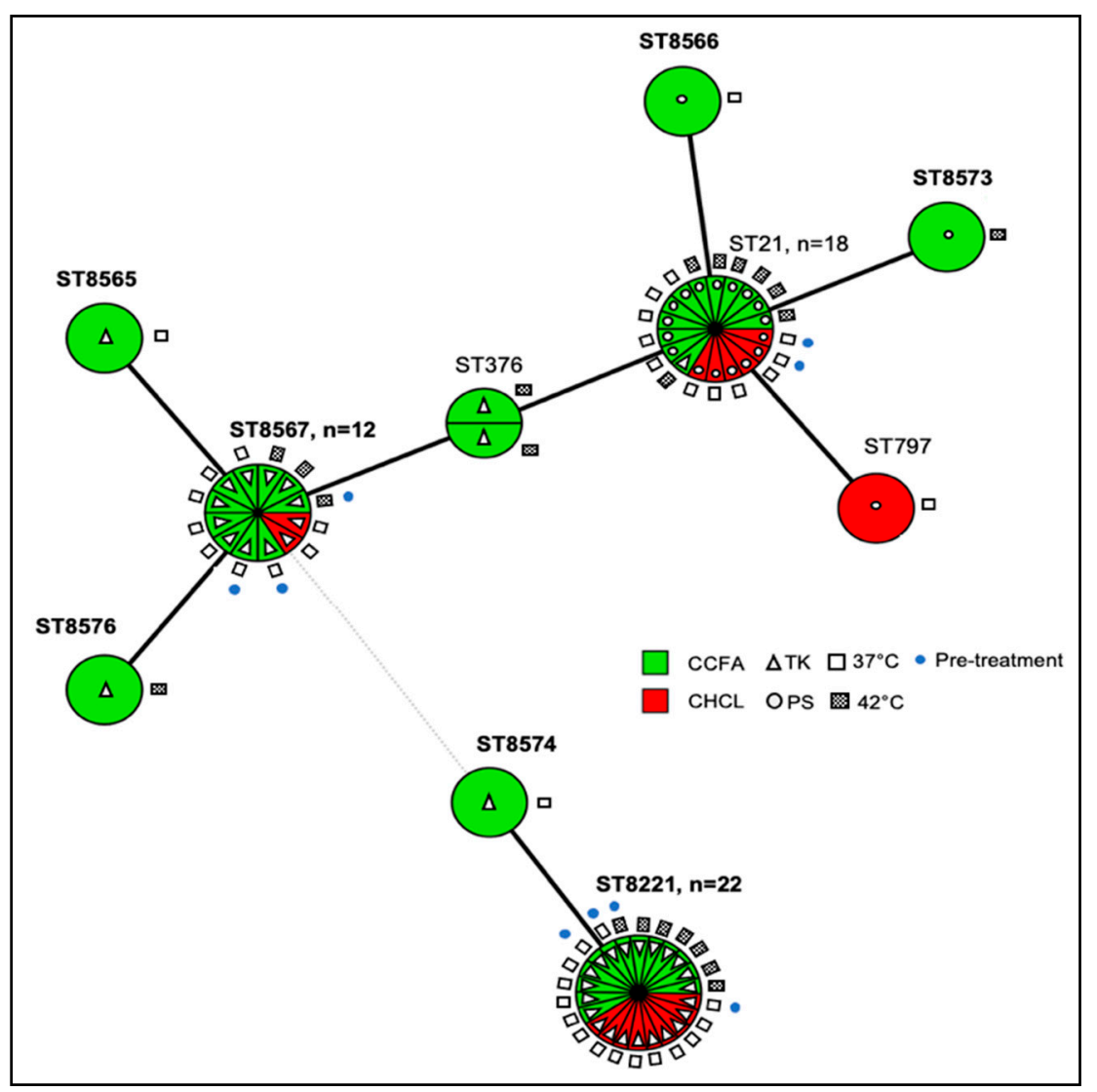

Figure 5. Minimum spanning tree (MST) demonstrating multilocus sequence typing (MLST)-based sequence types (STs) in C. jejuni from the CCFA and CHCL treatment groups. Each ST is represented by a circle, and partitions within each circle represent individual isolates with the respective ST. Circles without partitions (e.g., ST-797) indicate STs encountered only once. Green and red indicate the CCFA and CHCL group, respectively. AMR profile abbreviations: TK (rectangles), resistance only to tetracycline and kanamycin; PS (circles), pan-susceptible. Open and gray rectangles represent isolates obtained at $37^{\circ} \mathrm{C}$ and $42{ }^{\circ} \mathrm{C}$, respectively, and blue dots represent isolates recovered pretreatment. Solid lines connect STs with a single-locus difference. The dashed line represents three or more allele differences, i.e., a different clonal complex (CC). STs above the dashed line belong to CC21, while the two STs below the dashed line belong to CC61. Novel STs are in bold. MLST analysis and MST construction were as described in Materials and Methods.

Three STs, i.e., ST-21 (CC21) and the two novel STs 8567 (CC21) and 8221 (CC61), markedly predominated in both the CCFA and the CHCL isolates and were encountered both pre- and post-treatment in each group (Figure 5). In addition, they were detected in isolates obtained at both $37^{\circ} \mathrm{C}$ and $42{ }^{\circ} \mathrm{C}$ (Figure 5). Overall, a greater number of STs were noted among the CCFA than the CHCL isolates (9 vs. 4, respectively). Of the six STs found 
only in the CCFA group, three, i.e., ST-376 and the novel STs 8573 and 8576, were only identified in isolates obtained at $42{ }^{\circ} \mathrm{C}$ (Figure 5). Nevertheless, even at $37^{\circ} \mathrm{C}$ the CCFA group isolates $(n=21)$ exhibited three novel STs (STs 8565, 8566, and 8574) unique to this treatment group, all among the post-treatment isolates, while no novel STs unique to the CHCL group were identified among the 18 isolates from the CHCL treatment (Figure 5). The novel STs identified post-treatment in the CCFA group were closely related to other predominant STs recovered from the animals. Thus, STs 8573 and 8566 had single-allele differences from ST-21; ST-8574 also differed from ST-8221 by one allele; and STs 8565 and 8576 had one-allele differences from ST-8567 (Figure 5). Collectively, the findings suggest that CCFA treatment may be associated with higher genotypic diversity, but potential impacts of treatment on diversity cannot be statistically assessed due to a lack of power from the limited number of isolates.

Isolates with the same AMR profile tended to have the same ST, with the sole exception of one C. jejuni strain of ST-21 which was resistant to kanamycin and tetracycline, while all other ST-21 isolates were pan-susceptible (Figure 5). A similar correlation between STs and AMR profile was noted with C. hyointestinalis, for which all isolates of ST-6 were resistant to tetracycline and nalidixic acid, while those of ST-41 were resistant only to nalidixic acid (Table S2).

\section{Discussion}

The bacterial killing effect of ceftiofur depends upon the duration of the exposure at levels exceeding the MIC [50]. As described before, CCFA persisted approximately 2-fold longer than CHCL in the colon [17]. Nevertheless, the observed colonic concentrations of ceftiofur are unlikely to inhibit C. jejuni, as Campylobacter is intrinsically resistant to most cephalosporins [51], and the MIC of ceftiofur sodium (10-20 $\mu \mathrm{g} / \mathrm{mL})$ was at least 7.5-fold higher than the highest concentration of ceftiofur measured in the colon $(1.32 \mu \mathrm{g} / \mathrm{mL})$. Therefore, the stable prevalence of $C$. jejuni-positive samples in the CCFA group in week 1 (Figure 3) may be due to CCFA causing a steady inhibitory effect on other ceftiofur-susceptible microbes in the colon. On the other hand, the fluctuating prevalence of C. jejuni-positive samples in the CHCL group in week 1 (Figure 3) may be due to the three consecutive injections of this antibiotic, with concomitant changes in colonic CHCL concentrations and the associated effects on ceftiofur-susceptible microbes. The steady CCFA treatment effect was accompanied with a significantly higher estimated prevalence of C. jejuni and Campylobacter spp. in the CCFA group than in the CHCL group during week 1 (Figure 2).

Administration of CCFA resulted in a significant $(p=0.0108)$ decrease in the estimated prevalence of Campylobacter spp. from week 1 to week 2, while the estimated prevalence of Campylobacter spp. from steers receiving CHCL was also lower in week 2 than in week 1 , though not significantly different. It is tempting to speculate that the reductions in the colonic drug concentrations led to Campylobacter spp. becoming outcompeted in the gut by the returning ceftiofur-susceptible bacteria. This is supported by previously-reported analyses of these samples which showed that in the CCFA group the mean concentration of $E$. coli decreased significantly within the first $48 \mathrm{~h}$ after treatment but increased slowly thereafter (over the subsequent 12 days of monitoring), while significant impacts on E. coli concentrations were not noted with CHCL treatment [17]. Metagenomic analysis has also revealed shifts in community composition of the dairy cow microbiome following ceftiofur treatment, specifically increases in Bacterioidia and decreases in Actinobacteria [16].

A previous study predicted that the intestinal ceftiofur equivalent concentration in 12-month steers after administration of CCFA would be approximately $5 \times$ higher than the detected peak colonic concentration of CCFA in this study $(0.43 \mu \mathrm{g} / \mathrm{mL})$ [52]. This deviation suggests the importance of determining the drug concentration at the site of action [17]. However, noticeable standard deviation may result from individual steer differences, as depicted in Figure 2. 
The primary Campylobacter species isolated from cattle feces in this study was C. jejuni. The significantly higher estimated prevalence of $C$. jejuni at $42{ }^{\circ} \mathrm{C}$ may reflect the fact that this is the optimal incubation temperature for "thermophilic" campylobacters such as C. jejuni, while several other Campylobacter species, including C. hyointestinalis and C. fetus, prefer $37^{\circ} \mathrm{C}[53,54]$. C. fetus and C. hyointestinalis from the CCFA samples that were processed at both $37^{\circ} \mathrm{C}$ and $42^{\circ} \mathrm{C}$ were indeed primarily recovered from $37^{\circ} \mathrm{C}$, suggesting that including this temperature would be preferable to adequately capture Campylobacter species diversity.

C. jejuni is inherently resistant to cephalosporins, including ceftiofur [51,55-57], but the potential impact of ceftiofur treatment on MIC of this antimicrobial for C. jejuni has not been reported before. Our study suggested that the MIC of ceftiofur for C. jejuni was not impacted by treatment with either of the two ceftiofur formulations. In contrast, the ceftiofur MIC for E. coli from the steers in the CCFA and CHCL groups reached peak values at $96 \mathrm{~h}$ (37.16 and $51.05 \mu \mathrm{g} / \mathrm{mL}$, respectively) and then returned to baseline at 14 days [17]. Singer et al. also found ceftiofur-resistant E. coli only during and immediately following ceftiofur treatment of dairy cattle [58], and a transient increase in ceftiofur-resistant E. coli was also noted upon treatment of feedlot cattle with CCFA [59].

The extent to which ceftiofur treatment may favor C. jejuni with specific AMR profiles, such as the TK profile observed here, remains poorly understood. In our study, animals in both trials appeared to be already colonized with C. jejuni TK before treatment but the prevalence of $C$. jejuni TK was higher post-CHCL treatment than post-CCFA treatment. Further studies with larger numbers of samples pre- and post-treatment will be needed to elucidate the potential impacts of ceftiofur treatment on specific genotypes and AMR profiles of Campylobacter spp. in cattle.

Among the ten C. jejuni STs that were identified in this study, two (STs 21 and 376) were reported previously. ST-21 has been commonly recovered from human samples [60-63], and in the PubMLST Campylobacter database ST-21 has been mainly reported in the UK (58.1\%, 2367/4073), with 13.0\% (531/4073) identified in the US. ST-376 isolates were mainly from the UK $(n=4)$ and have not been previously reported in the US. Our MLST analysis suggested that CCFA treatment may favor genotypic diversity. Several of the novel STs identified post-treatment in the CCFA animals were closely related to predominant STs identified in the study, suggesting that the diversification may have taken place within the animals during the study period. Factors that may favor such genetic diversification remain to be elucidated. Previous studies suggest that CCFA exerts greater impact on other microbiota in the gut than CHCL [17], which may also facilitate the propagation of Campylobacter and generation of diverse STs.

\section{Conclusions}

Studies investigating the impact of ceftiofur on Campylobacter in cattle have been lacking. Here, we found a transient but significant increase in the estimated prevalence of Campylobacter within one week after CCFA treatment of steers, as well as a trend for higher genotypic diversity of $C$. jejuni than observed upon CHCL treatment. Neither treatment was found to impact the MIC of ceftiofur and other tested antimicrobials for C. jejuni from the feces of the steers. Considering the lower likelihood of a temporary increase in Campylobacter prevalence and lower genotypic diversity, CHCL may be preferable. However, the higher prevalence of $C$. jejuni with concurrent resistance to tetracycline and kanamycin post-CHCL treatment than post-CCFA treatment merits further investigation. Considering the dynamic occurrence of Campylobacter in the individual steer, and the diverse Campylobacter species, STs, and AMR profiles, larger sample sizes and more pretreatment sampling points would be needed for further assessments of the impacts of ceftiofur treatment of cattle on Campylobacter spp. prevalence, diversity, and AMR profiles. To enhance recovery of the diverse Campylobacter species and genotypes, such future studies may benefit from applying both $37^{\circ} \mathrm{C}$ and $42{ }^{\circ} \mathrm{C}$ to isolate Campylobacter spp. from the bovine samples. 
Supplementary Materials: The following are available online at https://www.mdpi.com/2076 $-2607 / 9 / 2 / 318 / s 1$, Table S1: Isolate data, Table S2: Campylobacter spp. analyzed by multilocus sequence typing in this study, Table S3: Antimicrobial minimum inhibitory concentration (MIC) test of representative isolates.

Author Contributions: Conceptualization, D.F. and S.K.; methodology, D.F. and S.K.; formal analysis, J.O., S.F., and W.G.M.; investigation, S.F.; resources, D.F., W.G.M., and S.K.; writing-original draft preparation, S.F.; writing-review and editing, S.F., S.K., W.G.M., D.F., and J.O.; supervision, D.F. and S.K.; project administration, D.F. and S.K.; funding acquisition, D.F. and S.K. All authors have read and agreed to the published version of the manuscript.

Funding: This research was partially funded by the USDA National Institute of Food and Agriculture, awards 1010130, 1007631, and 2018-67017-27927.

Institutional Review Board Statement: This study was conducted under the approval of the NCSU Institutional Animal Care and Use Committee (protocol \# 18-020A).

Informed Consent Statement: Not applicable.

Data Availability Statement: All the data in this manuscript were in the Supplementary Tables.

Acknowledgments: We thank Jeffrey Niedemeyer, Victor Oladimeji Jayeola, and Phillip Brown for technical support. We thank all members of our laboratory for feedback and support.

Conflicts of Interest: The authors declare no conflict of interest. The funders had no role in the design of the study; in the collection, analyses, or interpretation of data; in the writing of the manuscript, or in the decision to publish the results.

\section{References}

1. Rushton, J.; Ferreira, J.P.; Stärk, K.D.C. Antimicrobial Resistance: The Use of Antimicrobials in the Livestock Sector; OECD Publishing: Paris, France, 2014; Volume 68. [CrossRef]

2. Marshall, B.M.; Levy, S.B. Food animals and antimicrobials: Impacts on human health. Clin. Microbiol. Rev. 2011, 24, 718-733. [CrossRef] [PubMed]

3. Oliver, S.P.; Murinda, S.E.; Jayarao, B.M. Impact of antibiotic use in adult dairy cows on antimicrobial resistance of veterinary and human pathogens: A comprehensive review. Foodborne Pathog. Dis. 2011, 8, 337-355. [CrossRef]

4. FDA. 2018 Summary Report on Antimicrobials Sold or Distributed for Use in Food-Producing Animals. Available online: https:/ / www.fda.gov/media/133411/download (accessed on 11 December 2020).

5. USDA APHIS VS CEAH NAHMS. Dairy 2014: Health and Management Practices on U.S. Dairy Operations. 2014. Available online: https:/ / www.aphis.usda.gov/animal_health/nahms/dairy/downloads / dairy14/Dairy14_dr_PartIII.pdf (accessed on 11 December 2020).

6. WHO. WHO List of Critically Important Antimicrobials for Human Medicine (WHO CIA List). Available online: https: / / www.who.int/foodsafety/publications/cia2017.pdf?ua=1 (accessed on 11 December 2020).

7. USDA APHIS VS CEAH NAHMS. Feedlot 2011: Part IV: Health and Health Management on U.S. Feedlots with a Capacity of 1000 or More Head. Available online: https:/ / www.aphis.usda.gov/animal_health/nahms/feedlot/downloads/feedlot2011/ Feed11_dr_PartIV_1.pdf (accessed on 6 January 2021).

8. USDA: APHIS VS:CEAH. Dairy 2007: Part II: Changes in the U.S. Dairy Cattle Industry, 1991-2007. Available online: https: //naldc.nal.usda.gov / download/38398/PDF (accessed on 6 January 2021).

9. USDA APHIS VS CEAH NAHMS. Dairy 2014: Milk Quality, Milking Procedures, and Mastitis on U.S. Dairies. 2014. Available online: https: / / www.aphis.usda.gov/animal_health/nahms/dairy/downloads / dairy14/Dairy14_dr_Mastitis.pdf (accessed on 6 January 2021).

10. Pereira, R.V.; Siler, J.D.; Ng, J.C.; Davis, M.A.; Grohn, Y.T.; Warnick, L.D. Effect of on-farm use of antimicrobial drugs on resistance in fecal Escherichia coli of preweaned dairy calves. J. Dairy Sci. 2014, 97, 7644-7654. [CrossRef] [PubMed]

11. FDA; HHS. New Animal Drug; Cephalosporins Drugs; Extralabel Animal Drug Use; Order of Prohibition. Fed. Regist. 2012, 77, 735-745.

12. Salmon, S.A.; Watts, J.L.; Yancey, R.J., Jr. In vitro activity of ceftiofur and its primary metabolite, desfuroylceftiofur, against organisms of veterinary importance. J. Vet. Diagn. Investig. 1996, 8, 332-336. [CrossRef]

13. Haggett, E.F.; Wilson, W.D. Overview of the use of antimicrobials for the treatment of bacterial infections in horses. Equine Vet. Educ. 2008, 20, 433-448. [CrossRef]

14. U.S. National Library of Medicine. EXCEDE—Ceftiofur Injection, Suspension. Available online: https://dailymed.nlm.nih.gov/ dailymed/archives/fdaDrugInfo.cfm?archiveid=187510 (accessed on 11 December 2020).

15. U.S. National Library of Medicine. EXCENEL RTU STERILE-Ceftiofur Hydrochloride Injection, Suspension. Available online: https:/ / dailymed.nlm.nih.gov / dailymed/archives/fdaDrugInfo.cfm?archiveid=188500 (accessed on 11 December 2020). 
16. Chambers, L.; Yang, Y.; Littier, H.; Ray, P.; Zhang, T.; Pruden, A.; Strickland, M.; Knowlton, K. Metagenomic analysis of antibiotic resistance genes in dairy cow feces following therapeutic administration of third generation cephalosporin. PLOS ONE 2015, 10, e0133764. [CrossRef]

17. Foster, D.M.; Jacob, M.E.; Farmer, K.A.; Callahan, B.J.; Theriot, C.M.; Kathariou, S.; Cernicchiaro, N.; Prange, T.; Papich, M.G. Ceftiofur formulation differentially affects the intestinal drug concentration, resistance of fecal Escherichia coli, and the microbiome of steers. PLoS ONE 2019, 14, e0223378. [CrossRef]

18. Gorden, P.J.; Kleinhenz, M.D.; Wulf, L.W.; Rajewski, S.J.; Wang, C.; Gehring, R.; Coetzee, J.F. Comparative plasma and interstitial fluid pharmacokinetics of flunixin meglumine and ceftiofur hydrochloride following individual and co-administration in dairy cows. J. Vet. Pharmacol. Ther. 2018, 41, 76-82. [CrossRef]

19. Hornish, R.E.; Kotarski, S.F. Cephalosporins in veterinary medicine-Ceftiofur use in food animals. Curr. Top. Med. Chem. 2002, 2, 717-731. [CrossRef]

20. Agga, G.E.; Schmidt, J.W.; Arthur, T.M. Antimicrobial-resistant fecal bacteria from ceftiofur-treated and nonantimicrobial-treated comingled beef cows at a cow-calf operation. Microb. Drug Resist. 2016, 22, 598-608. [CrossRef]

21. Dutil, L.; Irwin, R.; Finley, R.; Ng, L.K.; Avery, B.; Boerlin, P.; Bourgault, A.M.; Cole, L.; Daignault, D.; Desruisseau, A.; et al. Ceftiofur resistance in Salmonella enterica serovar Heidelberg from chicken meat and humans, Canada. Emerg. Infect. Dis. 2010, 16, 48-54. [CrossRef]

22. Dunne, E.F.; Fey, P.D.; Kludt, P.; Reporter, R.; Mostashari, F.; Shillam, P.; Wicklund, J.; Miller, C.; Holland, B.; Stamey, K.; et al. Emergence of domestically acquired ceftriaxone-resistant Salmonella infections associated with AmpC beta-lactamase. JAMA 2000, 284, 3151-3156. [CrossRef]

23. Apley, M.D.; Coetzee, J.F. Antimicrobial Drug Use in Cattle. In Antimicrobial Therapy in Veterinary Medicine, 5th ed.; Giguère, S., Prescott, J.F., Dowling, P.M., Eds.; John Wiley \& Sons, Inc.: Hoboken, NJ, USA, 2013; pp. 495-518. [CrossRef]

24. Zoetis. AIF Product Comparison. Available online: https://www.zoetisus.com/dairy/avoidresidues/PDF/EXD13065_AIF_ ComparisonChart.pdf (accessed on 11 December 2020).

25. Foster, D.M.; Jacob, M.E.; Warren, C.D.; Papich, M.G. Pharmacokinetics of enrofloxacin and ceftiofur in plasma, interstitial fluid, and gastrointestinal tract of calves after subcutaneous injection, and bactericidal impacts on representative enteric bacteria. J. Vet. Pharmacol. Ther. 2016, 39, 62-71. [CrossRef]

26. Taylor, E.A.; Jordan, E.R.; Garcia, J.A.; Hagevoort, G.R.; Norman, K.N.; Lawhon, S.D.; Piñeiro, J.M.; Scott, H.M. Effects of two-dose ceftiofur treatment for metritis on the temporal dynamics of antimicrobial resistance among fecal Escherichia coli in Holstein-Friesian dairy cows. PLoS ONE 2019, 14, e0220068. [CrossRef]

27. Silva, J.; Leite, D.; Fernandes, M.; Mena, C.; Gibbs, P.A.; Teixeira, P. Campylobacter spp. as a foodborne pathogen: A review. Front. Microbiol. 2011, 2, 200. [CrossRef] [PubMed]

28. Wilson, D.J.; Gabriel, E.; Leatherbarrow, A.J.H.; Cheesbrough, J.; Gee, S.; Bolton, E.; Fox, A.; Fearnhead, P.; Hart, C.A.; Diggle, P.J. Tracing the source of campylobacteriosis. PLoS Genet. 2008, 6, e1000203. [CrossRef]

29. Tack, D.M.; Ray, L.; Griffin, P.M.; Cieslak, P.R.; Dunn, J.; Rissman, T.; Jervis, R.; Lathrop, S.; Muse, A.; Duwell, M.; et al. Preliminary incidence and trends of infections with pathogens transmitted commonly through food-Foodborne diseases active surveillance network, 10 U.S. sites, 2016-2019. Morb. Mortal. Wkly. Rep. 2020, 69, 509-514. [CrossRef]

30. Young, K.T.; Davis, L.M.; Dirita, V.J. Campylobacter jejuni: Molecular biology and pathogenesis. Nat. Rev. Microbiol. 2007, 5, 665-679. [CrossRef]

31. Bolinger, H.; Kathariou, S. The current state of macrolide resistance in Campylobacter spp.: Trends and impacts of resistance mechanisms. Appl. Environ. Microbiol. 2017, 83, e00416-17. [CrossRef]

32. CDC. Antibiotic Resistance Threats in the United States, 2019. Available online: https://www.cdc.gov/drugresistance/biggestthreats.html (accessed on 11 January 2021).

33. Ellis-Iversen, J.; Cook, A.J.; Smith, R.P.; Pritchard, G.C.; Nielen, M. Temporal patterns and risk factors for Escherichia coli O157 and Campylobacter spp. in young cattle. J. Food Prot. 2009, 72, 490-496. [CrossRef]

34. Epps, S.V.; Harvey, R.B.; Hume, M.E.; Phillips, T.D.; Anderson, R.C.; Nisbet, D.J. Foodborne Campylobacter: Infections, metabolism, pathogenesis and reservoirs. Int. J. Environ. Res. Public Health 2013, 10, 6292-6304. [CrossRef]

35. Davis, M.A.; Moore, D.L.; Baker, K.N.; French, N.P.; Patnode, M.; Hensley, J.; Macdonald, K.; Besser, T.E. Risk factors for campylobacteriosis in two Washington State counties with high numbers of dairy farms. J. Clin. Microbiol. 2013, 51, $3921-3927$. [CrossRef] [PubMed]

36. Friedman, C.R.; Hoekstra, R.M.; Samuel, M.; Marcus, R.; Bender, J.; Shiferaw, B.; Reddy, S.; Ahuja, S.D.; Helfrick, D.L.; Hardnett, F.; et al. Emerging Infections Program FoodNet Working Group. Risk factors for sporadic Campylobacter infection in the United States: A case-control study in FoodNet sites. Clin. Infect. Dis. 2004, 38, S285-S296. [CrossRef]

37. Davis, K.R.; Dunn, A.C.; Burnett, C.; McCullough, L.; Dimond, M.; Wagner, J.; Smith, L.; Carter, A.; Willardson, S.; Nakashima, A.K. Campylobacter jejuni infections associated with raw milk consumption-Utah, 2014. Morb. Mortal. Wkly. Rep. 2016, 65, 301-305. [CrossRef]

38. European Food Safety Authority; European Centre for Disease Prevention and Control. The European Union summary report on trends and sources of zoonoses, zoonotic agents and food-borne outbreaks in 2017. EFSA J. 2018, 16, e05500. [CrossRef]

39. Fernandes, A.M.; Balasegaram, S.; Willis, C.; Wimalarathna, H.M.; Maiden, M.C.; McCarthy, N.D. Partial failure of milk pasteurization as a risk for the transmission of Campylobacter from cattle to humans. Clin. Infect. Dis. 2015, 61, 903-909. [CrossRef] 
40. Davis, J.L.; Foster, D.M.; Papich, M.G. Pharmacokinetics and tissue distribution of enrofloxacin and its active metabolite ciprofloxacin in calves. J. Vet. Pharmacol. Ther. 2007, 30, 564-571. [CrossRef]

41. Ferguson, K.M.; Jacob, M.E.; Theriot, C.M.; Callahan, B.J.; Prange, T.; Papich, M.G.; Foster, D.M. Dosing regimen of enrofloxacin impacts intestinal pharmacokinetics and the fecal microbiota in steers. Front. Microbiol. 2018, 9, 2190. [CrossRef]

42. Jaglan, P.S.; Cox, B.L.; Arnold, T.S.; Kubicek, M.F.; Stuart, D.J.; Gilbertson, T.J. Liquid chromatographic determination of desfuroylceftiofur metabolite of ceftiofur as residue in cattle plasma. J. Assoc. Off. Anal. Chem. 1990, 73, 26-30. [CrossRef] [PubMed]

43. Gharst, G.; Hanson, D.; Kathariou, S. Effect of direct culture versus selective enrichment on the isolation of thermophilic Campylobacter from feces of mature cattle at harvest. J. Food Prot. 2006, 69, 1024-1027. [CrossRef]

44. Smith, K.; Reimers, N.; Barnes, H.J.; Lee, B.C.; Siletzky, R.; Kathariou, S. Campylobacter colonization of sibling turkey flocks reared under different management conditions. J. Food Prot. 2004, 67, 1463-1468. [CrossRef]

45. Wang, G.; Clark, C.G.; Taylor, T.M.; Pucknell, C.; Barton, C.; Price, L.; Woodward, D.L.; Rodgers, F.G. Colony multiplex PCR assay for identification and differentiation of Campylobacter jejuni, C. coli, C. lari, C. upsaliensis and C. fetus subsp. fetus. J. Clin. Microbiol. 2002, 40, 4744-4747. [CrossRef]

46. Miller, W.G.; Chapman, M.H.; Yee, E.; On, S.L.; McNulty, D.K.; Lastovica, A.J.; Carroll, A.M.; McNamara, E.B.; Duffy, G.; Mandrell, R.E. Multilocus sequence typing methods for the emerging Campylobacter species C. hyointestinalis, C. lanienae, C. sputorum, C. concisus, and C. curvus. Front. Cell. Infect. Microbiol. 2012, 2, 45. [CrossRef]

47. Miller, W.G.; Englen, M.D.; Kathariou, S.; Wesley, I.V.; Wang, G.; Pittenger-Alley, L.; Siletz, R.M.; Muraoka, W.; Fedorka-Cray, P.J.; Mandrell, R.E. Identification of host-associated alleles by multilocus sequence typing of Campylobacter coli strains from food animals. Microbiology 2006, 152, 245-255. [CrossRef] [PubMed]

48. Gu, W.; Siletzky, R.M.; Wright, S.; Islam, M.; Kathariou, S. Antimicrobial susceptibility profiles and strain type diversity of Campylobacter jejuni isolates from turkeys in eastern North Carolina. Appl. Environ. Microbiol. 2009, 75, 474-482. [CrossRef]

49. Niedermeyer, J.A.; Ring, L.; Miller, W.G.; Genger, S.; Lindsey, C.P.; Osborne, J.; Kathariou, S. Proximity to other commercial turkey farms affects colonization onset, genotypes, and antimicrobial resistance profiles of Campylobacter spp. in turkeys: Suggestive evidence from a paired-farm model. Appl. Environ. Microbiol. 2018, 84, e01212-e01218. [CrossRef]

50. Ambrose, P.G.; Bhavnani, S.M.; Rubino, C.M.; Louie, A.; Gumbo, T.; Forrest, A.; Drusano, G.L. Pharmacokineticspharmacodynamics of antimicrobial therapy: It's not just for mice anymore. Clin. Infect. Dis. 2007, 44, 79-86. [CrossRef]

51. Zhang, Q.; Plummer, P.J. Mechanisms of antibiotic resistance in Campylobacter. In Campylobacter, 3rd ed.; Nachamkin, I., Szymanski, C.M., Blaser, M.J., Eds.; American Society for Microbiology Press: Washington, DC, USA, 2008; pp. 263-276.

52. Volkova, V.V.; Lanzas, C.; Lu, Z.; Gröhn, Y.T. Mathematical model of plasmid-mediated resistance to ceftiofur in commensal enteric Escherichia coli of cattle. PLoS ONE 2012, 7, e36738. [CrossRef]

53. Wagenaar, J.A.; Van Bergen, M.A.; Blaser, M.J.; Tauxe, R.V.; Newell, D.G.; Van Putten, J.P. Campylobacter fetus infections in humans: Exposure and disease. Clin. Infect. Dis. 2014, 58, 1579-1586. [CrossRef]

54. On, S.; Miller, W.G.; Houf, K.; Fox, J.G.; Vandamme, P. Minimal standards for describing new species belonging to the families Campylobacteraceae and Helicobacteraceae: Campylobacter, Arcobacter, Helicobacter and Wolinella spp. Int. J. Syst. Evol. Microbiol. 2017, 67, 5296-5311. [CrossRef]

55. Thibodeau, A.; Fravalo, P.; Laurent-Lewandowski, S.; Guévremont, E.; Quessy, S.; Letellier, A. Presence and characterization of Campylobacter jejuni in organically raised chickens in Quebec. Can. J. Vet. Res. 2011, 75, 298-307.

56. Luangtongkum, T.; Jeon, B.; Han, J.; Plummer, P.; Logue, C.M.; Zhang, Q. Antibiotic resistance in Campylobacter: Emergence, transmission and persistence. Future Microbiol. 2009, 4, 189-200. [CrossRef] [PubMed]

57. Tajada, P.; Gomez-Graces, J.L.; Alós, J.I.; Balas, D.; Cogollos, R. Antimicrobial susceptibilities of Campylobacter jejuni and Campylobacter coli to 12 beta-lactam agents and combinations with beta-lactamase inhibitors. Antimicrob. Agents Chemother. 1996, 40, 1924-1925. [CrossRef]

58. Singer, R.S.; Patterson, S.K.; Wallace, R.L. Effects of therapeutic ceftiofur administration to dairy cattle on Escherichia coli dynamics in the intestinal tract. Appl. Environ. Microbiol. 2008, 74, 6956-6962. [CrossRef]

59. Lowrance, T.C.; Loneragan, G.H.; Kunze, D.J.; Platt, T.M.; Ives, S.E.; Scott, H.M.; Norby, B.; Echeverry, A.; Brashears, M.M. Changes in antimicrobial susceptibility in a population of Escherichia coli isolated from feedlot cattle administered ceftiofur crystalline-free acid. Am. J. Vet. Res. 2007, 68, 501-507. [CrossRef]

60. Cody, A.J.; McCarthy, N.D.; Jansen van Rensburg, M.; Isinkaye, T.; Bentley, S.D.; Parkhill, J.; Dingle, K.E.; Bowler, I.C.; Jolley, K.A.; Maiden, M.C. Real-time genomic epidemiological evaluation of human Campylobacter isolates by use of whole-genome multilocus sequence typing. J. Clin. Microbiol. 2013, 51, 2526-2534. [CrossRef]

61. De Haan, C.P.; Llarena, A.K.; Revez, J.; Hänninen, M.L. Association of Campylobacter jejuni metabolic traits with multilocus sequence types. Appl. Environ. Microbiol. 2012, 78, 5550-5554. [CrossRef] [PubMed]

62. Gripp, E.; Hlahla, D.; Didelot, X.; Kops, F.; Maurischat, S.; Tedin, K.; Alter, T.; Ellerbroek, L.; Schreiber, K.; Schomburg, D.; et al. Closely related Campylobacter jejuni strains from different sources reveal a generalist rather than a specialist lifestyle. $B M C$ Genomics 2011, 12, 584. [CrossRef] [PubMed]

63. Revez, J.; Zhang, J.; Schott, T.; Kivistö, R.; Rossi, M.; Hänninen, M.L. Genomic variation between Campylobacter jejuni isolates associated with milk-borne-disease outbreaks. J. Clin. Microbiol. 2014, 52, 2782-2786. [CrossRef] 